\title{
Blind Equalization of DS-CDMA and MC-CDMA Modulations In Time-Variant Frequency Selective Channels
}

\author{
Francesc Rey, Gregori Vázquez \\ Department of Signal Theory and Communications, Polytechnic University of Catalonia \\ UPC Campus Nord-Mòdul D5, c/ Jordi Girona 1-3, 08034 Barcelona (Spain) \\ Tel. +34-93-401.64.54 Fax. +34-93-401.64.47 \\ E-mail: \{frey, gregori\}@gps.tsc.upc.es
}

\begin{abstract}
The paper addresses the blind equalization problem of spread spectrum modulations in the presence of fast time-variant frequency selective channels. The basic assumption of the paper is that the channel response exhibit fast changes. A second goal of the paper is to force the definition of a universal CDMA blind equalization scheme that is capable to perform for DSCDMA or Multi-Carrier CDMA signal modulations without any modification in the equalizer. The formulation of the equalization scheme allows the consideration of temporal and/or spatial diversity frontend receivers. The result is a high performance system that uses a deterministic blind criterion to equalize the channel, avoiding the use of stochastic methods. The proposed technique performs direct channel equalization without previous channel estimation. Although the proposed equalizer in this work performs equalization at chip rate, this paper suggests a solution to achieve equalization at lower rates.
\end{abstract}

\section{INTRODUCTION}

Spread spectrum technology is in special interest when dealing with wireless fading channels because it has its own capabilities to combat the hostile channel effect. Nevertheless due to the frequency selectivity of the channel, the orthogonality between user codes at the receiver is lost, and some algorithms to combat this channel impact must be approached. This justifies that the equalization of the multipath transmission channel has recently received considerable attention.

The RAKE correlator is the optimum receiver for processing a spread spectrum signal, but it requires estimating the channel coefficients, which is not feasible if the channel is changing rapidly. Another algorithms are based in correlation techniques, but assume that the channel parameters change slowly considering a linear time invariant system in sufficient short intervals [1]. The best solution considering the time-variant nature of this kind of channels requires the

\footnotetext{
* This work has been partially supported by the Spanish Government (TIC96-0500-C10-01, TIC98-0412, TIC98-0703) and the Catalan government (CIRIT 1998SGR-00081).
}

use of deterministic methods, which make use of the a priori known signal structure, avoiding the use of conventional stochastic algorithms.

This paper is the natural extension of works done in [2] and [3], where equalizers based on a deterministic criterion were developed to directly identify the transmitted signal by means of spatial and/or temporal diversity. It proposes a mobile communications scheme that combines the use of CDMA systems with a deterministic blind equalizer. The result is a system that exploits the inherent structure of the transmitted data due to the spreading process, obtaining a robust equalization criterion against the frequency selectivity of the fading channel. The equalization process can be applied in single user and multiuser systems with the only restriction that the signal users must be synchronously received. In both cases the symbol detection is done in two steps, first the transmitted symbols are estimated (equalization process), and latter a single user or multiuser detector is required.

In special interest are spread spectrum equalizers that perform equalization at symbol rate. The main advantage of those equalizers is they do not work at high chip rates, a drawback when using long spreading code sequences. A design achieving equalization at lower rates is presented in [4]. Although the proposed equalizer in this work performs equalization at chip rate, it is suggested how to take into account the solution proposed in [4] reducing the equalization speed.

The next section summarizes briefly the signal model in a multiuser CDMA system. Section III justifies equalization design using the criterion suggested in [2] and [3], and obtains the proposed channel equalization algorithm. Single user and multiuser cases are separately studied. To conclude that section a solution to achieve equalization at rates lower than chip rate is proposed. Section IV derives the CRB covariance matrix for the transmitted symbol estimator in order to study the performance of algorithm. Finally section V presents some simulations results to illustrate the proposed scheme. 


\section{SIGNAL MODEL}

In a multiuser CDMA system several users can transmit simultaneously, so the received signal can be modeled as the summation of the $K$ users with additive channel noise:

$$
r(t)=\sum_{i=1}^{K} r^{i}(t)+n(t)
$$

where $n(t)$ is assumed to be white gaussian noise with zero mean and variance $\sigma_{w}{ }^{2}$.

For each user the received signal can be expressed as the convolution of the transmitted signal with the time-variant frequency selective channel $h(\tau, t)$.

$$
r^{k}(t)=h^{k}(\tau, t) * s^{k}(t)
$$

Finally the transmitted signal for each user $s^{k}(t)$ can be modeled as:

$$
s^{k}(t)=\sum_{n=0}^{N s-1} d_{n}^{k} \sum_{i=0}^{L c-1} c_{i}^{k} p\left(t-n T_{c}\right)
$$

where $d_{n}^{k} \in\{ \pm 1 \pm j\}$ are the k-user transmitted bits, $c_{i}{ }^{k}$ is the PN sequence, $L_{c}$ is the length of the spreading code, $p(t)$ is the chip pulse shaping and $T_{c}$ is the chip period.

Writing (3) in a matrix notation, a signal model, which can be identified with the model presented in [3], is obtained. This model is based in temporal and/or spatial diversity receivers. Thus the equalizer has $B$ samples ( $B$ is the diversity order) of the same transmitted information observed through different channels. Under the assumption that the $B$ channels have no common zeros, the perfect equalization of the frequency selective fading channels is allowed. Section III compares the results in [2] and [3] with the signal model proposed in this work and derives a criterion to design the equalizer scheme.

\section{PROBLEM STATEMENT}

This section obtains the equalization criterion that allows detecting the transmitted symbols in frequency selective fading channels. The proposed algorithm uses the matrix model derived from (3) applying the criterion proposed in [3], which is based on the insertion of certain structure in the transmitted information using a linear transformation. To simplify the notation let us first obtain the expressions for the single user model, and later we will generate the multiuser scheme.

\section{A. Single user}

If $\boldsymbol{d}$ is a vector that contains the $N_{s}$ information symbols:

$$
\boldsymbol{d}=\left[\begin{array}{llll}
d_{0} & d_{1} & \ldots & d_{N s-1}
\end{array}\right]^{T}
$$

considering a rectangular pulse for $p(t)$, and sampling the signal at $T s=T c$ (one sample per chip), the spreading can be modeled by means of a matrix as:

$$
\boldsymbol{G}_{\boldsymbol{c}}=\left[\begin{array}{cccc}
\boldsymbol{c} & \boldsymbol{0}_{L c} & \ldots & \boldsymbol{0}_{L c} \\
\boldsymbol{0}_{L c} & \boldsymbol{c} & \ldots & \boldsymbol{0}_{L c} \\
\vdots & \vdots & \ddots & \vdots \\
\boldsymbol{0}_{L c} & \boldsymbol{0}_{L c} & \ldots & \boldsymbol{c}
\end{array}\right]
$$

where $c$ is the spreading code

$$
\mathbf{c}=\left[\begin{array}{llll}
c_{0} & c_{1} & \ldots & c_{L c-1}
\end{array}\right]^{T}
$$

and $\boldsymbol{\theta}_{L c}$ is a $L_{c} \times 1$ zero vector.

Thus the spreaded sequence $s$ can be seen as the result of a linear transformation by means of the spreading matrix $\boldsymbol{G}_{\boldsymbol{c}}$ over the transmitted data $\boldsymbol{d}$ :

$$
s=G_{c} d
$$

The spreading matrix $G_{c}$ is a $\left(G_{p} x N_{s}\right) x N s$ column full rank matrix that provides structure to the encoded vector $s$. The transformation defines a signal subspace $S$, spanned by the $N_{s}$ columns of matrix $\boldsymbol{G}_{c}$, where vector $s$ is contained.

Accordingly it is possible to define an orthogonal subspace $S^{\perp}$ spanned by an $r=(G p-1) x N_{s}$ dimensional orthogonal basis and obtain its associated check matrix $\boldsymbol{G}_{c}{ }^{\perp}$. By definition this matrix accomplishes:

$$
G_{c}^{\perp} G_{c} d=G_{c}^{\perp} s=0
$$

The check matrix will detect changes between the transmitted code and the received information. The outputs of the marginal channels $C^{i}(i=1,2 \ldots B)$ to the transmitted data $s$, and the channel noise contributions $\boldsymbol{w}^{i}$ will force the received data to be contained in $S \oplus S^{\perp}$. Basically, the projection of the received data in the orthogonal subspace $S^{\perp}$ is used by the equalizer to characterize the noise and channel response.

Thus, the two equalizer design equations becomes [3]:

$$
\begin{aligned}
& \hat{\boldsymbol{s}}=\boldsymbol{Y}_{\boldsymbol{t}} \boldsymbol{e} \\
& \boldsymbol{G}_{c}^{\perp} \hat{\boldsymbol{s}}=G_{c}^{\perp} \boldsymbol{Y}_{\boldsymbol{t}} \boldsymbol{e}=\mathbf{0}
\end{aligned}
$$

In (9) $e$ is the vector that contains the equalizer coefficients, and $Y_{t}$ are the first $n$ rows of the convolution matrix $Y$ that contains the symbols at the output of the channel.

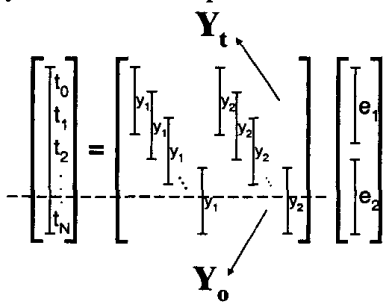

Thus an estimation of the transmitted vector $s$ is obtained in the first equation. The second equation contains information on the residual ISI and noise in the orthogonal signal subspace $S^{\perp}$, and might be zero if a perfect equalization is accomplished. 
According to (9), the equalizer coefficients can be obtained by means of a cost function that maximizes the signal to noise+ISI ratio in the orthogonal subspace (SNIR):

$$
\operatorname{SINR}=\frac{e^{H} Y_{t}^{H} Y_{t} e}{e^{H} Y_{t}^{H} G_{c}^{\perp H} G_{c}^{\perp} Y_{t} e}
$$

and the equalizer that maximizes (10) corresponds to the generalized eigenvector associated with the maximum generalized eigenvalue:

$$
Y_{t}^{H} Y_{t} e=\lambda_{\max } Y_{t}^{H} C_{c}^{\perp H} G_{c}^{\perp} Y_{t} e
$$

\section{B. Multiuser}

Defining $d^{k}$ as the symbol vector for user $k$ (eq. 4 ) and $\boldsymbol{G}_{c}{ }^{k}$ the spreading matrix for user $k$ (eq. 5), the spreaded sequence can be modeled in a matrix notation as (without lost of generality the 2 users case is considered):

$$
s=s^{1}+s^{2}=\underbrace{\left[\begin{array}{ll}
G_{c}^{1} & G_{c}^{2}
\end{array}\right]}_{G c}\left[\begin{array}{l}
d^{1} \\
d^{2}
\end{array}\right]
$$

In the multiuser case the spreading matrix is a column full rank matrix ( $\left.G_{p} x N_{s}\right) x K N_{s}$ and thus the equalization scheme can be used again.

Let us now study how the perfect equalization criterion [3] is accomplished in the multiuser case. Focusing on $k$ user let us denote $C^{k, i}$ as the Sylvester matrix that contains the $i$-channel coefficients, where $i$ is the diversity branch $i=1$...B. Thus equation 8 can be written as:

$$
y^{i}=\left[\begin{array}{lll}
C^{1, i} & \ldots & C^{K, i}
\end{array}\right]\left[\begin{array}{c}
s^{1} \\
\vdots \\
s^{K}
\end{array}\right]
$$

where $y^{i}$ is the vector at the output of the $i$-channel.

Combining all branches, the equalizer output is given by:

$$
r=\underbrace{\left[\begin{array}{lll}
S^{1} & \ldots & S^{K}
\end{array}\right]}_{S}\left[\begin{array}{ccc}
C^{11} & \ldots & C^{I B} \\
\vdots & \ddots & \vdots \\
C^{K 1} & \ldots & C^{K B}
\end{array}\right]\left[\begin{array}{c}
e^{1} \\
\vdots \\
e^{B}
\end{array}\right]
$$

where $S^{k}$ is the Sylvester matrix whose columns are shifted versions of the $k$-user transmitted samples $s k$.

Finally to design the equalizer coefficients satisfying the second equation in (9) means:

$$
\begin{aligned}
G_{c}^{\perp} Y_{t} e & =G_{c}^{\perp} S_{t} C e \\
& =\left[\begin{array}{llllllll}
0 & p_{2}^{1} & \ldots & p_{L+\nu-1}^{1} & 0 & p_{2}^{2} & \ldots & p_{L+\nu-1}^{2}
\end{array}\right] C e=0
\end{aligned}
$$

where $S_{t}=S(1: n$,$) is by definition a sub-matrix of S$ matrix composed by rows $l$ to $n$, such that for each user the first columns is the transmitted vector $s^{k}$ (orthogonal to $G_{c}^{\perp}$ ).

If the column vectors $\left\{\boldsymbol{p}_{i}^{k}\right\} \quad i=2, \ldots \mathrm{L}+v-1$ are linearly independent, the solution for equation (15) is equivalent to the perfect equalization for each user:

$$
C e=\left[\begin{array}{llllllll}
\beta^{1} & 0 & \ldots & \beta^{2} & 0 & \ldots & \beta^{K} & \ldots
\end{array}\right]^{T}
$$

where $\beta^{k}$ is an arbitrary multiplicative complex constant for user $k$. Thus at the output of the equalizer:

$$
\boldsymbol{r}=\sum_{i=1}^{K} \beta^{i} \boldsymbol{s}^{i}
$$

To force the column vectors in (15) to be linearly independent means that the minimum required redundancy for perfect channel equalization becomes:

$$
\text { Redundancy }=\operatorname{rank}\left(G_{c}^{\perp} S_{t}\right) \geq K(L+v-2)
$$

Furthermore (16) has solution if there are equal or lower equations than variables, and thus:

$$
v \geq \frac{K(L-1)}{B-K}
$$

where $K$ is the number of users, $L$ the length of the channel, $B$ the diversity order and $v$ the equalizer length for each branch. Equation (18) forces a new condition in the length of the equalizer design.

To conclude this section let us suggest how the proposed equalizer can be modified to achieve equalization at lower than chip rates. Figure 1 presents the scheme in [4] allowing that. In those case the despreading is done before the equalization process, equalizing the equivalent channel that includes spreading, frequency-selective channel distortion and despreading processes.

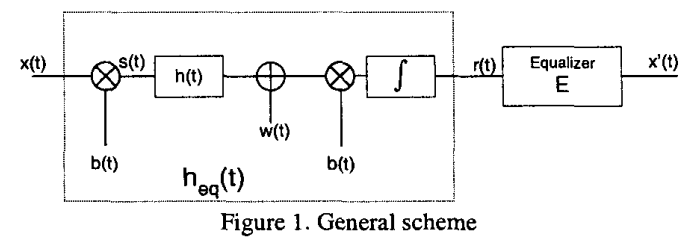

Notice that in the algorithm presented in this paper it is not possible to carry out despreading before equalization. That is because it assumes that the information vector at the output of the equalizer is still the spreaded information vector (9).

A solution to consider first the despreading, reducing the equalization rate, is to spread the information signal in two steps. In figure 1 , if signal $x(t)$ has been spreaded according to the structure required in (7), equation (9) can already been accomplished at the equalizer output. In such a case the spreading code is illustrated in the next figure:

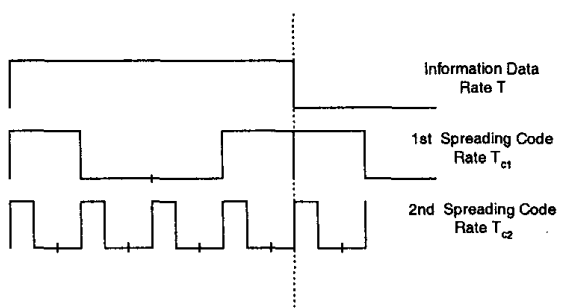

Figure 2.Two steps spreading process 
Where the second spreading code is used to achieve the spreading at the output of the channel (fig.1) while the first spreading code provide the structure to the transmitted data and can be used to perform the equalization (6).

\section{PERFORMANCE ANALISYS}

In this section we provide a performance analysis of our algorithm. The goal will be to derive the Cramér-Rao Bound (CRB) for the transmitted symbol estimator for user $k$. With the CRB we will obtain a lower bound to evaluate the mean-square error of the proposed scheme.

Following the same notation used in previous sections, $y^{i}$ is the vector at the output of the channel for the branch $i=1$...B. For user $k, C^{k, i}$ is the matrix containing the $i$ channel coefficients, and $s^{k}$ is the transmitted vector:

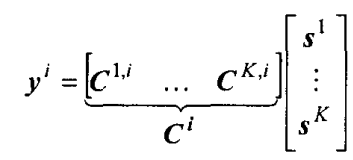

The likelihood function of the data is given by:

$$
f(y ; s)=\frac{1}{\left(\pi \sigma_{w}^{2}\right)^{N}} \exp \left\{-\frac{1}{\sigma_{w}^{2}} \sum_{i=1}^{B}\left(y^{i}-C^{i} s\right)^{H}\left(y^{i}-C^{i} s\right)\right\}
$$

Thus the log-likelihood function is:

$$
\ln [f(y ; s)]=\text { const }-\frac{1}{\sigma_{w}^{2}} \sum_{i=1}^{B}\left(y^{i}-C^{i} s\right)^{H}\left(y^{i}-C^{i} s\right)
$$

If we denote $\Delta$ as:

$$
\begin{aligned}
\Delta & =\frac{\partial}{\partial s^{k^{*}}} \ln [f(y ; s)] \\
& =\frac{1}{\sigma_{w}^{2}} \sum_{i=1}^{B} C^{k i}\left(y^{i}-C^{i} s\right)
\end{aligned}
$$

And the Fischer information matrix (FIM) for the symbol vector $s^{k}$ is given by:

$$
\begin{aligned}
\boldsymbol{J} & =E\left\{\boldsymbol{\Delta \Delta ^ { H }}\right\} \\
& =\left(\frac{1}{\sigma_{w}^{2}}\right)^{2} \sum_{i, j=1}^{B} E\left\{\boldsymbol{C}^{i^{H}}\left(y^{i}-C^{i} \boldsymbol{s}\right)\left(y^{j}-\boldsymbol{C}^{j} \boldsymbol{s}\right)^{H} \boldsymbol{C}^{j}\right\}
\end{aligned}
$$

The expression $\left(y^{i}-\boldsymbol{C} s\right)$ is the noise vector $\boldsymbol{w}^{i}$, and computing the expectation:

$$
E\left\{\boldsymbol{w}^{i} \boldsymbol{w}^{j^{H}}\right\}=\sigma_{w}^{2} \boldsymbol{I} \delta(i-j)
$$

we obtain :

$$
\boldsymbol{J}=\frac{1}{\sigma_{w}^{2}} \sum_{i=1}^{B} C^{k i H} C^{k i}
$$

And thus the CRB can be obtained directly from the FIM computing its inverse:

$$
\boldsymbol{C R B}=\boldsymbol{J}^{-1}=\sigma_{w}^{2}\left(\boldsymbol{C}^{k^{H}} \boldsymbol{C}^{k}\right)^{-1}
$$

where the matrix $C^{k}$ is defined as:

$$
\boldsymbol{C}^{k}=\left[\begin{array}{lll}
C^{k 1} & \ldots & C^{k B}
\end{array}\right]^{T}
$$

The derived $\mathrm{CRB}$ do not considers that some parametric constraints can be considered on the estimated parameters. Equation (8) defines the constraints over $\boldsymbol{s}$, the estimated vector. Under these assumptions [5] and [6] (see also references) derived a CRB under parametric constraints: If the estimated parameters satisfies:

$$
f(s)=0
$$

And define the gradient matrix (assumed to have full row rank) of the constraints as:

$$
\boldsymbol{F}(\boldsymbol{s})=\frac{\partial f(\boldsymbol{s})}{\partial \boldsymbol{s}}
$$

the exists a matrix $U$ so that:

$$
F(s) U=0
$$

and the derived $\mathrm{CRB}$ is:

$$
\boldsymbol{C R B}=\boldsymbol{U}\left(\boldsymbol{U}^{H} \boldsymbol{J U}\right)^{-1} \boldsymbol{U}^{H}
$$

In our problem the previous matrix can be identified as:

$$
\begin{gathered}
F(s) \longrightarrow G_{c}^{\perp} \\
U \longrightarrow G_{c}
\end{gathered}
$$

\section{SIMULATIONS}

To illustrate the performance of the proposed algorithm and compare it with the analytical MSE expression obtained in section IV, some simulation examples are here presented.

In all cases the length of the transmitted frame information was 32 QPSK data symbols, the channel EbNo was $12 \mathrm{~dB}$ and the FIR filters in each equalizer branch had 8 coefficients. The order of the spatial and temporal diversity was $\mathrm{B}=6$. Finally the $\mathrm{PN}$ sequences considered were Gold codes of length 15 chips.

The frequency selective multipath channel responses were generated according to [7]:

$$
h(n)=\sum_{i=1}^{L} \alpha_{i} \delta(n-i)
$$

where $h(n)$ is the channel response, $L$ the length of the channel, in our case the channel length was five chips, and $\alpha_{i}$ the random complex value for path $i$.

Figure 2 shows the performance of the spatial diversity receiver; while figure 3 illustrates the temporal diversity one. In both cases simulations display the percentage of realizations (over 1000) for which the equalizer output EbNo was higher than the value indicated in the $\mathrm{x}$-axis. The results are focused on user 1 and several simulations were done increasing the number of users (maintaining the same channel for user 1). As it can be seen when the number of 
users increases the performance of the algorithm decreases because the number of channels to equalize is higher.

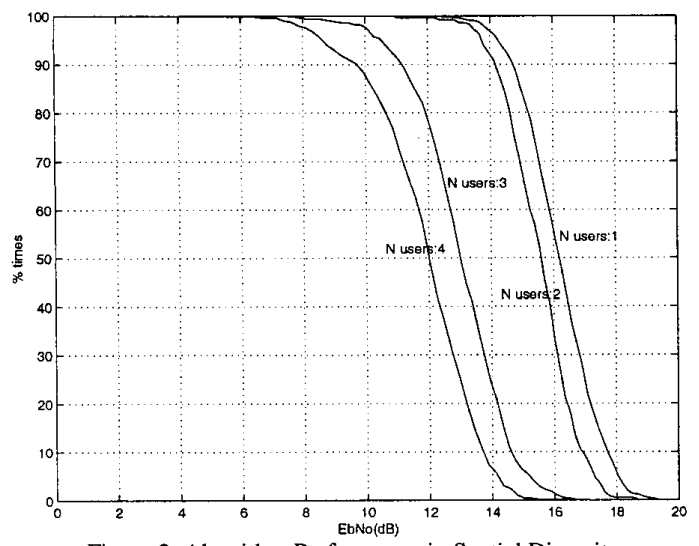

Figure 2. Algorithm Performance in Spatial Diversity. EbNo $=12 \mathrm{~dB}$. Nousers: $1-4$

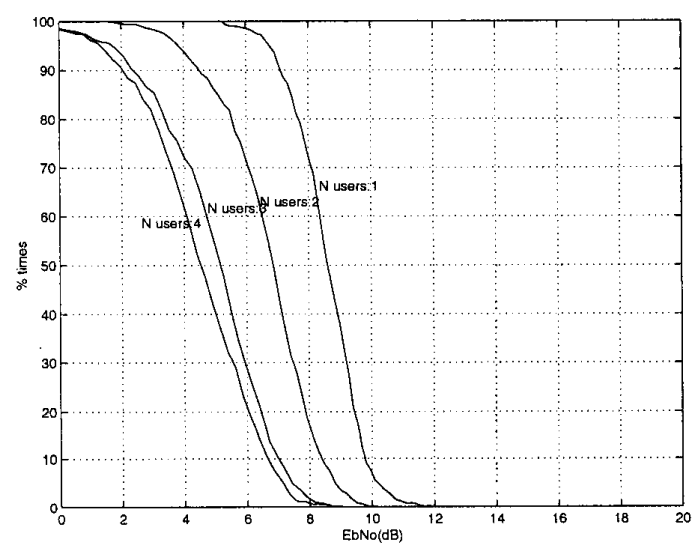

Figure 3. Algorithm Performance in Temporal Diversity $\mathrm{EbNo}=12 \mathrm{~dB}$. Nousers: 1.4

Notice that in the spatial diversity receiver, B sensors are considered, and so the maximum output EbNo could be:

$$
\max \left\{(E b N o)_{\text {out }}\right\}=(E b N o)_{\text {in }}+10 \log (B)
$$

Figure 4 compares the CRB derived in section IV with the normalized root-mean square error (RMSE) defined bellow:

$$
R M S E=\sqrt{\frac{1}{N_{t}} \sum_{i=1}^{N_{t}} \frac{\left\|\hat{d}_{i}-d_{i}\right\|^{2}}{\left\|d_{i}\right\|^{2}}}
$$

where $N_{t}$ is the number of Monte Carlo realizations (500). The RMSE is employed as a performance measure for the proposed equalizer based on spatial and temporal diversity receivers.

It is seen that the RMSE decreases as $1 / \mathrm{EbNo}$, as CRB does.

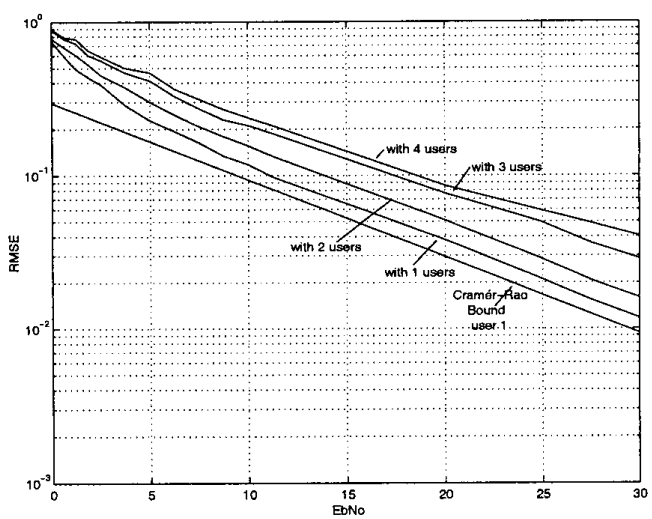

Figure 4. Root-Mean Square Error vs. EbNo

\section{CONCLUSIONS}

A blind equalization scheme for CDMA systems in multipath frequency selective fading channels has been introduced. The main contribution in this work is the exploitation of the inherent structure in the transmitted data due to orthogonality properties of the spreading codes. The spreading process allows defining an orthogonal subspace $S^{\perp}$, which is used in the receiver to obtain a robust equalization criterion against the frequency selective fading channels.

The resulting equalizer, applied over single user and multiuser CDMA systems, exploit the high capacity of blind algorithms based on block coded modulations avoiding the insertion of certain redundancy in the transmitter.

\section{REFERENCES}

[1] S.E. Bensley, B. Aazhang, 'Subspace-based channel estimation for code division multiple access communications Systems', IEEE Trans. Commun., August 1996.

[2] M. Lamarca, G. Vázquez, 'Diversity techniques for blind channel equalization in mobile communications', Proceedings of PIMRC'97, Helsinki (Finland).

[3] F. Rey, M. Lamarca, G. Vázquez, 'Redundancy in Block Coded Modulations for Channel Equalization Based on Spatial and Temporal Diversity', Proc. of ICASSP'99, Phoenix (USA).

[4] G. Vázquez, F. Rey, M. Lamarca, J.R. Fonollosa, 'Diversity in Mobile Communications for Blind Detection of Block-Coded Modulations', Proceedings of VTC'99, Houston (USA).

[5] P. Stoica,B. Chong, 'On the Cramér-Rao Bounds Under Parametric Constraints', IEEE Signal Processing Letters, Vol. 5, No 7 July 1998.

[6] H.Liu, G.Xu, 'Closed-form blind symbol estimation in digital communications', IEEE Trans. Signal Processing, vol. 43 Nov.1995.

[7] R. Steele, Mobile Radio Communications, Pentech Press. 